\title{
More ways than one for disorder
}

\section{Despite half a century's hard work, there remain intolerably few circumstances in which the thermo- dynamics of disordered states can be calculated from plausible models.}

\begin{abstract}
Wiтн every month that passes, it becomes more and more apparent that the transition between microscopically ordered and disordered states is of enormous technical as well as theoretical importance, yet people's capacity to handle problems in this field seems merely to inch forward. Collective ingenuity, never at rest, meanwhile continues to invent new problems for solution. The common but depressing escape seems to be the devising of ever more intricate numerical simulations of particular problems, no doubt with the best intentions that these will be suggestive of more general understanding. But the hope is most often frustrated.
\end{abstract}

To be fair to the field and its practitioners, the most obvious problems are those which are for good reasons the most difficult to solve. Take any phase transition, say between a non-polar liquid, such as butane, and its vapour. One might assume that the vapour is a perfect gas. but the condensed (and more ordered) phase is an exceedingly disordered aggregation of molecules that must differ intrinsically from the perfect gas by virtue of the manner in which neighbouring molecules interact with each other. To say the least, they cannot be microscopic billiard balls. The mere fact that surfaces form between the liquid and the gas phase shows that two-body interactions are insufficient for an explanation. People hoping to make sense of this order-disorder transition must plunge into a theory of liquids, with its Kirkwood clusters and the like.

That is why much hope has always centred on the use of simpler systems as models of real-life transitions. Liquids and vapours differ from each other not merely in their degree of orderliness but also by the degree to which the free energy of each phase is dominated by the kinetic energy and, for the liquid, by the multimolecular potential energy. For the study of order-disorder transitions, the ideal would obviously be a system in which there are two phases which are virtually identical except in their degree of order. What can such systems be?

Unhappily, the most obvious case, that of a ferromagnetic material, is far from easily handled. To be sure, the pre-conditions are readily satisfied. A ferromagnetic solid will seem much the same above and below the Curie temperature, so that experimental measurements, say of the specific heat, may be taken as direct measures of the energy involved in the transition from order to disorder. But even here direct comparison between experiment and some theoretical model is far from simple. In real ferromagnets, it is not possible to make the simplifying assumption that only interactions between nearest neighbours matter, while even if it were, the resulting simplified model would prove to be intractable.

So much has been demonstrated by the large numbers of those who, in the past several decades, have broken their heads on just that task. The simplest model of a ferromagnet is a cubic lattice with little dipole magnets at each vertex. Even with the simplifying assumptions that the magnets can point in only one direction or its opposite, that only nearest-neighbour dipoles interact and that the lattice does not vibrate, whatever its temperature, it is not possible to find an analytical expression for, say, the free energy of this system. Even though the two-dimensional analogue of this problem was solved half a century ago by Lars Onsager, and while there are endless complicating modifications of it, the simple cubic-lattice model of a ferromagnet remains intractable

That is why there must be great rejoicing that at least one class of problems. dealt with in the $1930 \mathrm{~s}$ by people such as H.A. Bethe, is still firmly within the field of tractability, that of the solid solutions capable of forming ordered states. Brass, the alloy of copper and zinc, is one of these. Even with the correct proportions, the solid solution is a soft material which. when annealed, becomes hard and useable.

The thermodynamics textbooks give good accounts of the solution of such problems, which historically seem to have been the basis for the powerful way of treating these problems developed in the Soviet Union by the late Lev Landau and his then protegée, V. L. Ginsburg. Choose an "order parameter" (in the case of brass, the proportion of atoms out of place), assume the free energy to be some polynomial function of this parameter, calculate its minimum and then relate that to the physics of the problem.

This technique has been applied in fields as different as classical superconductivity and the superfluidity of helium. It works. even though there may be some trouble in telling just what physical significance to attach to the order parameter. But it would be better to start at the other end of the problem - with a model which is a platusible representation of a physical system - and to be able to calcu- late the properties of the system from that

The general frustration that these problems are usually intractable no doubt explains why so many other approaches to order-disorder problems have now been followed. One approach is to enumerate the possible configurations of a system numerically, by means of some some mechanism dependent on the generation of random numbers. But the results remain obdurately numerical.

Much the same is true of attempts to calculate disordered structures such as the solid dendrites formed by crystallization from vapour or liquid. E. S. Langer was the first, two decades ago, to carry a macroscopic theory of a solidifying liquid onto a sufficiently microscopic scale to allow for processes which are limited by diffusion, but progress beyond that has been almost all numerical - E.T. Witten made a calculation, ten years ago, of diffusion-limited aggregation on a twodimensional lattice that has since become the prototype for the numerical calculation of fractal structures formed under a variety of circumstances.

There is a neat version of such a calculation, backed up by experiment. in the recent literature (Helgesen, G. et ol. Phys. Rev. Lett. 61. 1736; 1988). Using plastic microspheres loaded with iron oxide, this group has been able to show both experimentally and by calculation how the fractal dimensions of the aggregates formed change systematically with the magnetic moment and also with the strength of an externally applied field.

But this is only scratching at the outside surface of an important set of problems. On the face of things, for example, what is called high- $T$. superconductivity must crucially involve disorder of some kind. Departures from textbook stoichiometric compositions of the superconducting materials suggest disorder in the superconducting copper oxide layers. Similarly, the recent recognition of solid lattices with five- and ten-fold symmetry and the dependent issue of how these lattices grow by the aggregation of single atoms has provoked a series of questions intermediate between those raised by Witten and by the insoluble Ising lattice. And there is the whole string of problems involving macromolecules in gel formation. in diffusion through a gel and even in the transition from natural to denatured forms. It will be intolerable if all these problems remain only partly soluble for much longer.

John Maddox 